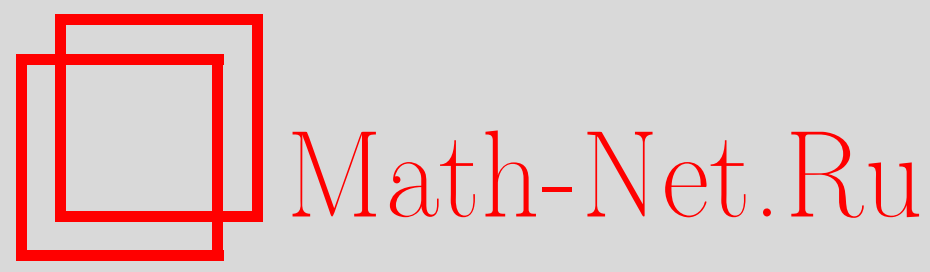

С. В. Алешин, Автоматное представление свободной группы, Дискрет. матем., 2011, том 23, выпуск 3, 32-56

DOI: https://doi.org/10.4213/dm1151

Использование Общероссийского математического портала Math-Net.Ru подразумевает, что вы прочитали и согласны с пользовательским соглашением http://www. mathnet.ru/rus/agreement

Параметры загрузки:

IP: 3.93 .64 .190

26 апреля 2023 г., 14:19:49 


\title{
Автоматное представление свободной группы
}

\author{
(c) 2011 г. С. В. Алешин
}

Построен новый пример свободной подгруппы группы автоматных подстановок. Указаны два автомата с тремя состояниями, которые порождают эту подгруппу, при этом внутренние полугруппы этих автоматов не являются группами.

Первый пример свободной подгруппы группы автоматных подстановок был предложен в [3], полное доказательство этого результата было получено в [4]. Автоматы из этого примера являются дважды групповыми - у них самих и обратных к ним автоматов внутренние полугруппы являются группами. Такие автоматы составляют малую долю всех автоматов. Более того, если рассматривать группы, порожденные инициальными подавтоматами одного автомата, то, как показано в [6], существует 122 неизоморфных группы, порожденных автоматами с тремя состояниями, и только автомат из [3] порождает свободную группу. В [5] указана автоматная группа без кручения (не свободная) с тремя образующими полугрупповыми автоматами.

Мы построим свободную группу, порожденную двумя полугрупповыми автоматами. Элементами группы автоматных подстановок $A S_{2}[1,2]$ являются взаимно-однозначные отображения множества слов в алфавите $\{0,1\}$, которые реализуются инициальными автоматами с входным и выходным алфавитом $\{0,1\}$. Рассмотрим два таких автомата $A$ и $B$, каждый с тремя состояниями. Диаграмма автомата $A$ приведена на рис. 1 ; как обычно, в круге, соответствующем состоянию, мы пишем номер состояния и подстановку на входном алфавите, которая реализуется в этом состоянии. В начальном состоянии 1 реализуется отрицание $\bar{x}-$ цикл $(0,1)$, в состояниях 2 и 3 реализуются тождественные подстановки $x$.

Отображение автомата $A$ обозначим через $f$. Обратное отображение $f^{-1}$ реализуется инициальным автоматом $\bar{A}$, приведенным на рис. 2 .

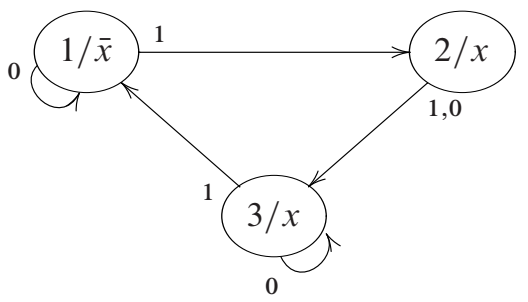

Pис. 1. 


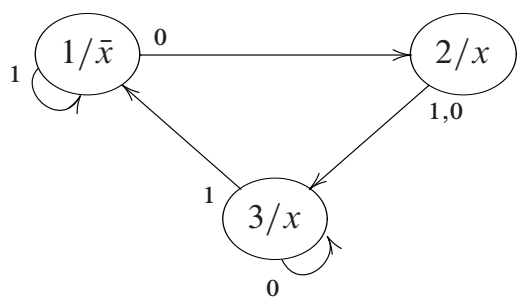

Рис. 2.

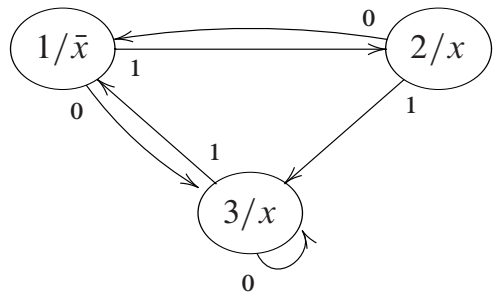

Pис. 3.

С использованием стандартных обозначений можно представить автомат $A$ как

$$
A=\{(0,1),(1,2,3),\{0,1\}, \varphi, \psi, 1\},
$$

где функция переходов есть

$$
\varphi: \quad \varphi(1,0)=1 ; \quad \varphi(1,1)=2 ; \quad \varphi(2,0)=\varphi(2,1)=3 ; \quad \varphi(3,0)=3 ; \quad \varphi(3,1)=1,
$$

а функция выходов есть

$$
\psi(1, x)=\bar{x} ; \quad \psi(2, x)=x ; \quad \psi(3, x)=x .
$$

Автомат $B$ представлен на диаграмме, приведенной на рис. 3. Его отображение обозначим через $h$. Обратное отображение $h^{-1}$ реализует автомат $\bar{B}$, приведенный на рис. 4 .

Ниже будет показано, что подгруппа $S A$ группы $S A_{2}$, порожденная элементами $\left\{f, f^{-1}, h, h^{-1}\right\}$, порождается ими свободно.

Если элементы $g_{1}, g_{2}$ группы $S A_{2}$ реализуются автоматами

$$
\begin{aligned}
& C_{1}=\left(\{0,1\}, Q_{1},\{0,1\}, \varphi_{1}, \psi_{1}, q_{1}^{\circ}\right), \\
& C_{2}=\left(\{0,1\}, Q_{2},\{0,1\}, \varphi_{2}, \psi_{2}, q_{2}^{\circ}\right),
\end{aligned}
$$

соответственно, то произведение $g=g_{1} g_{2}$ реализуется автоматом $C$, который является последовательным соединением $C_{1}$ и $C_{2}$, таким, что выход $C_{1}$ соединен с входом $C_{2}$, и $C=\left(\{0,1\}, Q,\{0,1\}, \varphi, \psi, q^{\circ}\right)$, где $Q \subseteq Q_{1} \times Q_{2}$;

$$
\begin{aligned}
& \varphi\left(\left(q_{1}, q_{2}\right), x\right)=\left(\varphi_{1}\left(q_{1}, x\right), \varphi_{2}\left(q_{2}, \psi_{1}\left(q_{1}, x\right)\right)\right) ; \\
& \psi\left(\left(q_{1}, q_{2}\right), x\right)=\psi_{2}\left(q_{2}, \psi\left(q_{1}, x\right)\right) .
\end{aligned}
$$




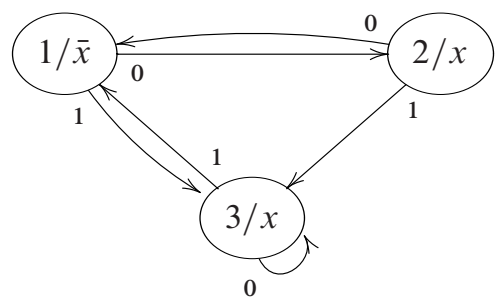

Pис. 4.

Множество состояний произведения $Q$ - это множество пар $\left(q_{1}, q_{2}\right), q_{1} \in Q_{1}$, $q_{2} \in Q_{2}$, которые достижимы из начального состояния $\left(q_{1}^{\circ}, q_{2}^{\circ}\right)$. Вообще говоря, разные пары из $Q$ могут соответствовать эквивалентным (неотличимым) состояниям автомата $C$. Например, произведение $f \cdot f^{-1}$ реализуется автоматом с одним состоянием - тождественное отображение $(0,1)^{*}$ на себя (единица группы $\left.A S_{2}\right)$, при этом множество пар, достижимых из начальной пары $(1,1)$, состоит из трех пар $(1,1),(2,2)$ и $(3,3)$.

Слову $R=r_{1} r_{2} \ldots r_{s}$ в алфавите $\left\{f, f^{-1}, h, h^{-1}\right\}$ соответствует произведение отображений $r_{1} r_{2} \cdots r_{s}$, которое реализуется автоматом, получающимся соединением автоматов из набора $\{A, \bar{A}, B, \bar{B}\}$. Слово $R$ называется правильным, если в нем не встречаются стоящие рядом $f$ и $f^{-1}$ или $h$ и $h^{-1}$. Наша задача - показать, что всякое правильное слово задает отображение, отличное от единицы группы $S A_{2}$.

Пусть

$$
\begin{aligned}
& C=(\{0,1\}, Q,\{0,1\}, \varphi, \psi), \\
& D=\left(\{0,1\}, P,\{0,1\}, \varphi^{\prime}, \psi^{\prime}\right)
\end{aligned}
$$

- приведенные автоматы, то есть у каждого из них состояния попарно отличимы.

Скажем, что автомат $C$ гомоморфен автомату $D$, если на множестве состояний $Q$ задано разбиение $\rho$ на непересекающиеся классы такое, что число классов равно числу состояний $D$, и существует отображение $v$ множества $Q$ на множество $P$ такое, что если $q_{1}$ и $q_{2}$ принадлежат одному классу разбиения $\rho$, то $v\left(q_{1}\right)=v\left(q_{2}\right)$ и $v(\varphi(q, x))=$ $\varphi^{\prime}(v(q), x)$ для любых $q \in Q, x \in\{0,1\}$.

Такой гомоморфизм называют также гомоморфизмом переходных систем.

Скажем, что автомат $F=(\{0,1\}, H,\{0,1\}, \hat{\varphi}, \hat{\psi})$ является подавтоматом автомата $C=(\{0,1\}, Q,\{0,1\}, \varphi, \psi)$, если $H \subseteq Q$, функции $\hat{\varphi}$ и $\hat{\psi}-$ ограничения функций $\varphi$ и $\psi$, соответственно, на множество $H$, и $H$ замкнуто относительно действия $\hat{\varphi}$, то есть $\varphi(q, x) \in H$, если $q \in H, x \in\{0,1\}$.

Скажем, что автомат $D$ делит автомат $C$, если найдется подавтомат автомата $C$, который гомоморфен автомату $D$.

Определим $S$-свойство слова.

Определение 1. Пусть $R=r_{1} r_{2} \ldots r_{s}-$ слово в алфавите $\left\{f, f^{-1}, h, h^{-1}\right\}$ и $r \in\left\{f, f^{-1}, h, h^{-1}\right\}, r \neq r_{1}$. Скажем, что слово $R$ обладает $S$-свойством, если автомат, реализующий элемент $r$, делит приведенный автомат, реализующий слово $R$.

Пусть $D, G$-автоматы с двумя состояниями, переходные системы которых приведены на рис. 5 . 
$D:$

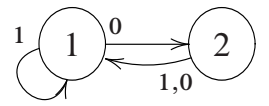

$G:$

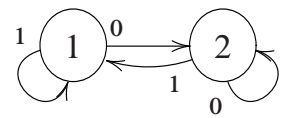

Рис. 5.

Лемма 1. Если $R=r_{1} r_{2} \ldots r_{s}-$ правильное слово, обладающее $S$-свойством, то для любого $t \geqslant 1$ имеет место один из случаев:

(а) слово $r_{t} r_{t+1} \ldots r_{s}$ обладает $S$-свойством,

(б) автомат D делит автомат, реализующий $r_{t} r_{t+1} \cdots r_{s}$,

(в) автомат $G$ делит автомат, реализующчий $r_{t} r_{t+1} \cdots r_{s}$.

Доказательство. Рассмотрим все возможные случаи.

Случай 1: $r_{1}=f, r=f^{-1}$. Пусть $L-$ приведенный автомат, реализующий отображение $r_{2} r_{3} \cdots r_{s}$. Тогда автомат, реализующий отображение $r_{1} r_{2} \cdots r_{s}$, есть суперпозиция автоматов $A$ и $L$, где

$$
L=\left(\{0,1\}, Q,\{0,1\}, \Phi, \psi, q_{\circ}\right) .
$$

Состояния суперпозиции могут быть заданы в виде пар $(i, q), i \in\{1,2,3\}, q \in Q$, при этом разные пары могут задавать неотличимые состояния. Обозначим приведенный автомат, реализующий произведение $r_{1} r_{2} \cdots r_{s}$ через $F$. По условию леммы, существует гомоморфизм $v$ из подавтомата $F$ на автомат $\bar{A}$. Этот гомоморфизм можно распространить на множество пар $(i, q)$, считая, что если пара $(i, q)$ задает состояние $u$ автомата $F$, то $v(i, q)=v(u)$. Гомоморфизм $v$ разбивает множество состояний подавтомата $F$ на три класса $K_{1}, K_{2}, K_{3}$ так, что $v\left(K_{s}\right)=s, s=1,2,3$. Множество пар, задающих состояния суперпозиции $A L$, также разобьется на три класса, при этом пары, задающие одно и то же состояние, окажутся в одном классе - пары из класса $R_{s}$ задают состояния из класса $K_{s}, s=1,2,3, R_{s} \cap R_{k}=\varnothing$ при $s \neq k$.

Обозначим через $R_{i s}$ множество пар вида $(i, q) \in R_{s}$. Функция переходов $\gamma$ суперпозиции $A L$ такова, что

$$
\gamma((i, q), x)=(\varphi(i, x), \Phi(q, \psi(i, x))),
$$

где $\varphi, \psi-$ функции переходов и выходов автомата $A$. Поэтому для классов $R_{i s}$ справедлива диаграмма переходов, приведенная на рис. 6. Для примера рассмотрим переход $R_{11} \stackrel{0}{\rightarrow} R_{12}$. В самом деле, для любой пары вида $(1, q) \gamma((1, q), 0)=\left(1, q^{\prime}\right)$ для некоторого $q^{\prime} \in Q$. Кроме того, для любой пары $(i, q) \in R_{1}$ имеет место равенство $v((i, q))=1$, и, так как $v$ - гомоморфизм,

$$
v(\gamma((i, q), 0))=\bar{\varphi}(v((i, q)), 0)=\bar{\varphi}(1,0)=2,
$$

где $\bar{\varphi}-$ функция переходов $\bar{A}$. Поэтому под действием входной буквы 0 любая пара из $R_{11}$ перейдет в некоторую пару из $R_{12}$. 


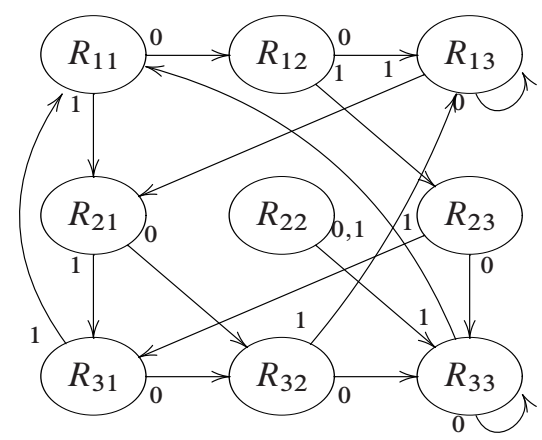

Рис. 6.

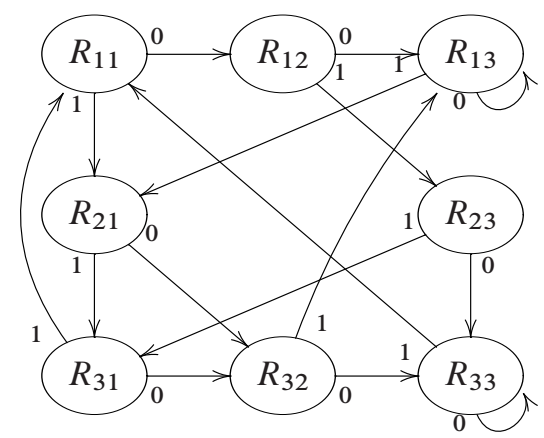

Рис. 7.

Так как никакая пара из $R_{22}$ не достижима из начальной пары, окончательно диаграмма принимает вид, приведенный на рис. 7.

Обозначим через $Q_{i s} \subseteq Q$ множество состояний автомата $L$ таких, что $q \in Q_{i s}$, если $(i q) \in R_{i s}$. Напомним, что выходная функция $\psi$ автомата $A$ такова, что

$$
\psi(1, x)=\bar{x}, \quad \psi(2, x)=\psi(3, x)=x .
$$

Поэтому для множеств $Q_{i s}$ состояний автомата $L$ имеет место диаграмма переходов, приведенная на рис. 8.

Для примера, пусть пара $(1, q) \in R_{11}$, тогда $q \in Q_{11}$. Если $\Phi-$ функция переходов $L$, то в суперпозиции $A L$

$$
\gamma((1, q), 0)=\left(1, q^{\prime}\right)=(\varphi(1,0), \Phi(\psi(q, 0), 1))=(1, \Phi(q, 1))=\left(1, q^{\prime}\right)
$$

то есть

$$
q^{\prime}=\Phi(q, 1)
$$

при этом $\left(1, q^{\prime}\right) \in R_{12}$ и $q^{\prime} \in Q_{12}$.

Для дальнейшего важно, что для всякого $i=1,2,3$ имеет место равенство $Q_{i m} \cap Q_{i n}=\varnothing$ при $m \neq n$. В самом деле, пусть $\left(i, q_{1}\right) \in R_{i m},\left(i, q_{2}\right) \in R_{i n}$. Тогда состояния $u_{1}, u_{2}$, задаваемые, соответственно, этими парами, отличимы, и значит отображения 


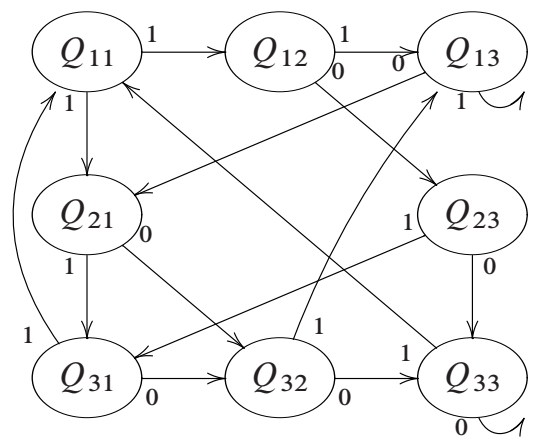

Pис. 8.

$F_{1}, F_{2}$, реализуемые автоматом $A L$ с начальными состояниями $u_{1}, u_{2}$, соответственно, различны, то есть

$$
F_{1}=A_{i} \cdot L_{q_{1}} \neq A_{i} \cdot L_{q_{2}},
$$

где $A_{i}$ - отображение автомата $A$ с начальным состоянием $i, L_{q}$ - отображение автомата $L$ с начальным состоянием $q$. Так как $A_{i}-$ взаимно однозначное отображение, то $L_{q_{1}} \neq L_{q_{2}}$, и $q_{1}, q_{2}$ отличимы.

Вообще говоря, классы $Q_{i m}$ и $Q_{j n}$ для разных $i, j$ могут пересекаться. Однако в данном случае мы покажем, что и для разных $i, j$ имеет место равенство $Q_{i m} \cap Q_{j n}=\varnothing$, тем самым система классов $\left\{Q_{i s}, i, s=1,2,3\right\}$, - это разбиение множества состояний автомата на непересекающиеся классы.

Если под действием сигнала $a$ состояния из множества $M_{1}$ переходят в состояния из множества $M_{2}$, то будем обозначать это как $M_{1} \stackrel{a}{\rightarrow} M_{2}$. Из диаграммы на рис. 8 видно, что

$$
\left(Q_{11} \cap Q_{21}\right) \stackrel{1}{\rightarrow}\left(Q_{12} \cap Q_{31}\right) \stackrel{1}{\rightarrow}\left(Q_{13} \cap Q_{11}\right)=\varnothing,
$$

и $Q_{12} \cap Q_{31}=\varnothing$. Далее,

$$
\left(Q_{11} \cap Q_{31}\right) \stackrel{1}{\rightarrow}\left(Q_{12} \cap Q_{31}\right)=\varnothing,
$$

поэтому $Q_{11} \cap Q_{23}=\varnothing$ и так далее. Используя диаграмму на рис. 8 , построим диаграмму пересечений классов, представленную на рис. 9. Здесь цифра, помещенная в два различных класса, обозначает множество состояний, равное пересечению этих классов. Например, цифра 1 обозначает пересечение классов $Q_{11} \cap Q_{21}$, цифра 2 - пересечение $Q_{11} \cap Q_{23}$ и так далее.

Имеем переходы для пересечений

$$
\begin{array}{llll}
1 \stackrel{1}{\rightarrow} 8 \stackrel{1}{\rightarrow} \varnothing, & 2 \stackrel{1}{\rightarrow} 8=\varnothing, & 3 \stackrel{1}{\rightarrow} \varnothing, & 4 \stackrel{0}{\rightarrow} 18 \stackrel{0}{\rightarrow} \varnothing, \\
5 \stackrel{1}{\rightarrow} \varnothing, & 6 \stackrel{1}{\rightarrow} 13 \stackrel{1}{\rightarrow} \varnothing, & 7 \stackrel{1}{\rightarrow} 13=\varnothing, & 8 \stackrel{1}{\rightarrow} \varnothing, \\
9 \stackrel{0}{\rightarrow} 21 \stackrel{1}{\rightarrow} 3=\varnothing, & 10 \stackrel{1}{\rightarrow} \varnothing, & 11 \stackrel{1}{\rightarrow} 13=\varnothing, & 12 \stackrel{1}{\rightarrow} 13=\varnothing, \\
14 \stackrel{0}{\rightarrow} 18=\varnothing, & 15 \stackrel{1}{\rightarrow} \varnothing, & 16 \stackrel{1}{\rightarrow} 3=\varnothing, & 17 \stackrel{0}{\rightarrow} \varnothing, \quad 18 \stackrel{0}{\rightarrow} \varnothing, \\
19 \stackrel{0}{\rightarrow} \varnothing, & 20 \stackrel{1}{\rightarrow} 13=\varnothing, & 21 \stackrel{1}{\rightarrow} 3=\varnothing,
\end{array}
$$




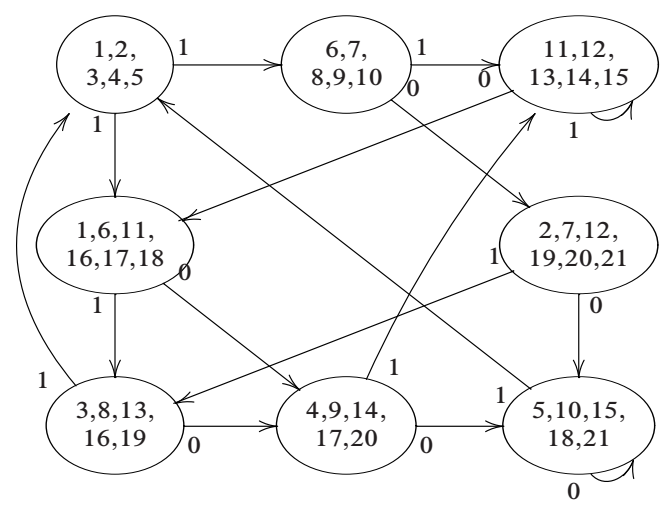

Рис. 9.

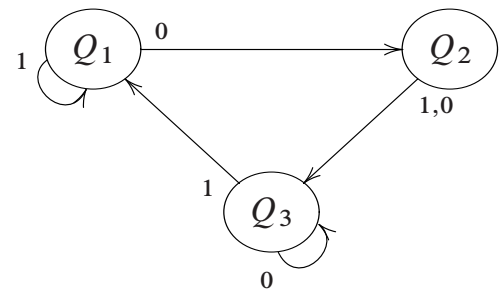

Рис. 10.

Таким образом, все пересечения пусты, и система классов $Q_{i s}-$ разбиение множества состояний автомата $L$. Поэтому и система классов $\left(Q_{1}, Q_{2}, Q_{3}\right), Q_{i}=U_{s} Q_{i s}$, образует разбиение множества состояний автомата $L$. Диаграмма переходов для классов $Q_{1}, Q_{2}$, $Q_{3}$ имеет вид, приведенный на рис. 10.

Она, очевидно, совпадает с диаграммой переходов автомата $\bar{A}$. Следовательно, автомат $L$ гомоморфен автомату $\bar{A}$. Слово $r_{2} r_{3} \ldots r_{s}$ не начинается с буквы $f^{-1}$, так как $r_{1} r_{2} \ldots r_{s}$ - правильное слово, и $r_{1}=f$. Таким образом, слово $r_{2} r_{3} \ldots r_{s}$ обладает $S$-свойством, и случай 1 , где $r_{1}=f, r=f^{-1}$, рассмотрен.

Поскольку граф диаграммы пересечений совпадает с графом диаграммы для классов $Q_{i s}$, в дальнейшем мы не будем предъявлять диаграмму $Q_{i s}$, а только диаграмму пересечений.

Случай 2: $r_{1}=f, r=h^{-1}$. Сохраняя построения и обозначения предыдущего случая, получаем диаграммы классов $R_{i s}$ (рис.. 11) и пересечений (рис. 12).

Справедливы соотношения

$$
\begin{array}{lcl}
3 \stackrel{1}{\rightarrow} \varnothing & 2 \stackrel{1}{\rightarrow} 8 \stackrel{1}{\rightarrow} \varnothing & 4 \stackrel{1}{\rightarrow} \varnothing \quad 5 \stackrel{1}{\rightarrow} \varnothing \\
1 \stackrel{1}{\rightarrow} 10 \stackrel{0}{\rightarrow} 21 \stackrel{1}{\rightarrow} 3=\varnothing & 6 \stackrel{1}{\rightarrow} 5=\varnothing & 7 \stackrel{1}{\rightarrow} 3=\varnothing \\
9 \stackrel{1}{\rightarrow} \varnothing & 11 \stackrel{1}{\rightarrow} 15 \stackrel{1}{\rightarrow} \varnothing & 12 \stackrel{1}{\rightarrow} 13 \stackrel{0}{\rightarrow} 17 \stackrel{1}{\rightarrow} 15=\varnothing
\end{array}
$$


Рис. 11.
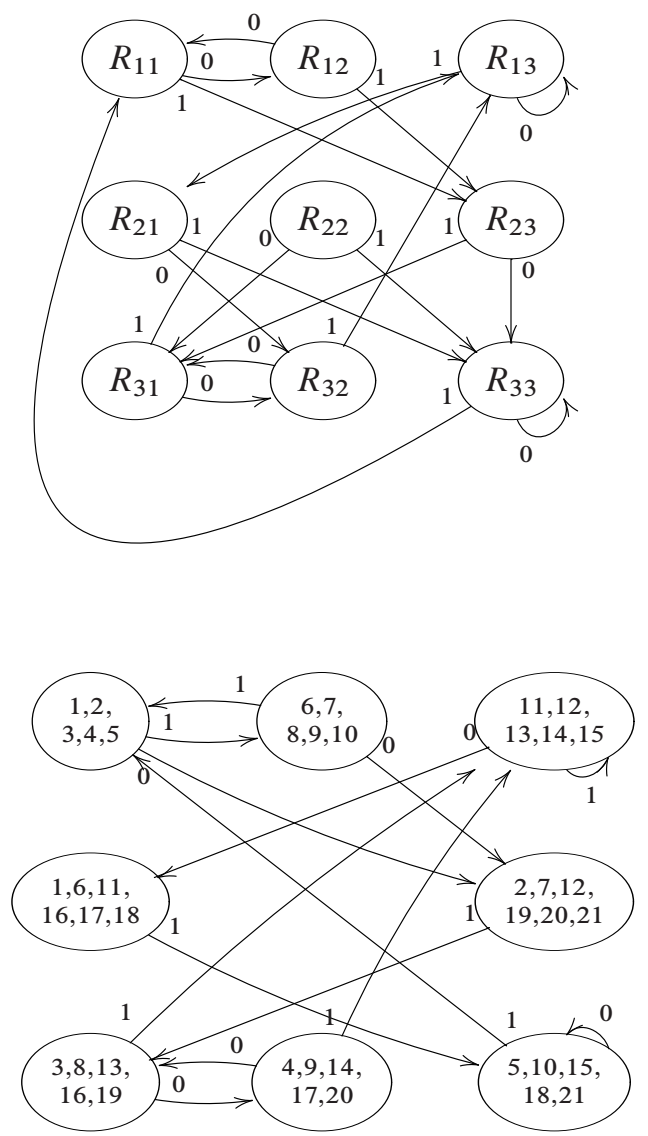

Рис. 12.

$$
\begin{aligned}
& 14 \stackrel{0}{\rightarrow} 16 \stackrel{1}{\rightarrow} 15=\varnothing \quad 18 \stackrel{1}{\rightarrow} 5=\varnothing \quad 19 \stackrel{1}{\rightarrow} 13=\varnothing \\
& 20 \stackrel{1}{\rightarrow} 13=\varnothing
\end{aligned}
$$

Таким образом, все пересечения классов пусты, поэтому тройка классов $\left(Q_{1}, Q_{2}, Q_{3}\right)$, $Q_{i}=\bigcup_{s} Q_{i s}$, образует разбиение множества состояний автомата $L$, а диаграмма этого разбиения совпадает с диаграммой автомата $\bar{A}$, и снова слово $r_{2} r_{3} \ldots r_{s}$ обладает $S$-свойством.

Случай 3: $r_{1}=f, r=h$. Диаграммы классов $R_{i s}$ (рис. 13) и диаграмма пересечений классов (рис. 14) дают соотношения

$$
1 \stackrel{1}{\rightarrow} 17 \stackrel{0}{\rightarrow} 19 \stackrel{1}{\rightarrow} 11 \stackrel{0}{\rightarrow} 25 \stackrel{1}{\rightarrow} 10 \stackrel{1}{\rightarrow} 1=\varnothing \quad 2 \stackrel{1}{\rightarrow} 18 \stackrel{1}{\rightarrow} \varnothing
$$


Рис. 13.
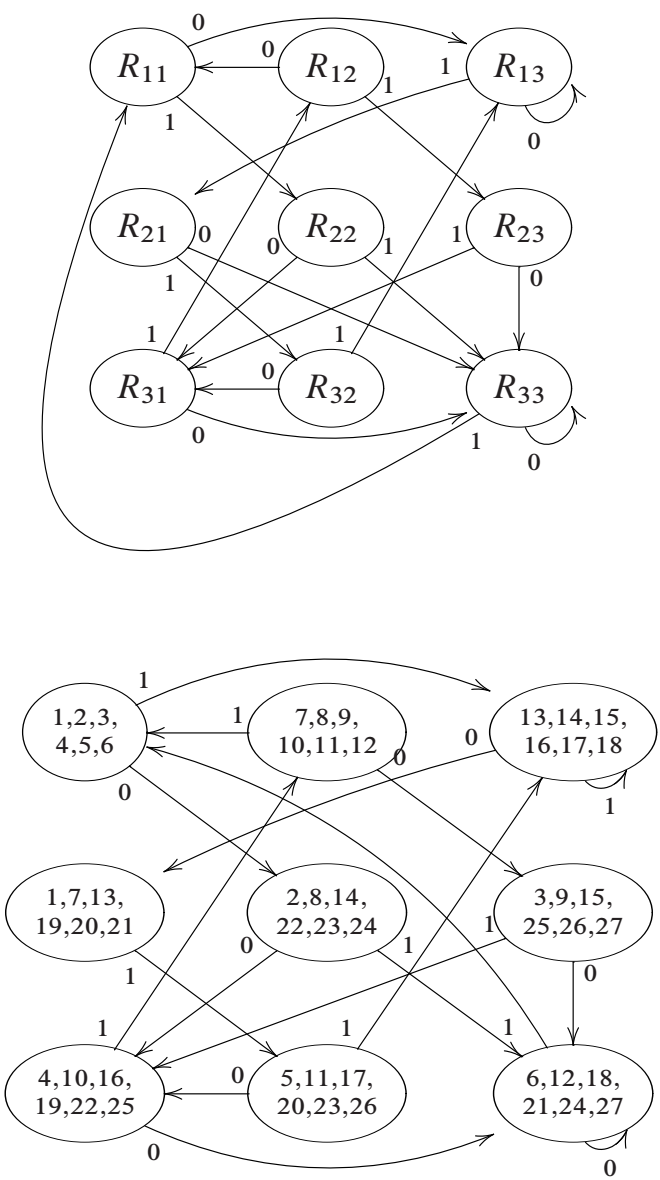

Рис. 14.

$$
\begin{aligned}
& 3 \stackrel{0}{\rightarrow} 24 \stackrel{0}{\rightarrow} \varnothing \quad 4 \stackrel{0}{\rightarrow} 24=\varnothing \quad 5 \stackrel{0}{\rightarrow} 22 \stackrel{0}{\rightarrow} \varnothing \quad 6 \stackrel{0}{\rightarrow} 24=\varnothing \\
& 7 \stackrel{1}{\rightarrow} 5=\varnothing \quad 8 \stackrel{1}{\rightarrow} 6=\varnothing \quad 9 \stackrel{1}{\rightarrow} 4=\varnothing \quad 12 \stackrel{0}{\rightarrow} 27 \stackrel{1}{\rightarrow} 4=\varnothing \\
& 13 \stackrel{1}{\rightarrow} 17=\varnothing \quad 14 \stackrel{1}{\rightarrow} 18=\varnothing \quad 15 \stackrel{0}{\rightarrow} 21 \stackrel{1}{\rightarrow} 5=\varnothing \quad 16 \stackrel{1}{\rightarrow} \varnothing \\
& 20 \stackrel{0}{\rightarrow} \varnothing \quad 22 \stackrel{1}{\rightarrow} \varnothing \quad 23 \stackrel{1}{\rightarrow} 18=\varnothing \quad 25 \stackrel{1}{\rightarrow} 10=\varnothing \quad 26 \stackrel{1}{\rightarrow} 16=\varnothing
\end{aligned}
$$

Таким образом, система классов $Q_{i s}$ - разбиение, система классов $\left(Q_{1}, Q_{2}, Q_{3}\right)-$ разбиение, диаграмма переходов для этой системы совпадает с диаграммой автомата $\bar{A}$, что и требовалось доказать.

Все случаи $r_{1}=f$ рассмотрены. Перейдем к случаям $r_{1}=f^{-1}$.

Случай 4: $r_{1}=f^{-1}, r=f$. Диаграмма классов $R_{i s}$ (рис. 15) и диаграмма пересечений классов (рис. 16) дают соотношения 


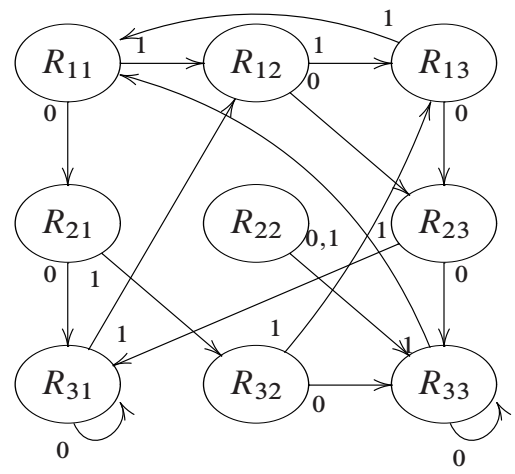

Рис. 15.

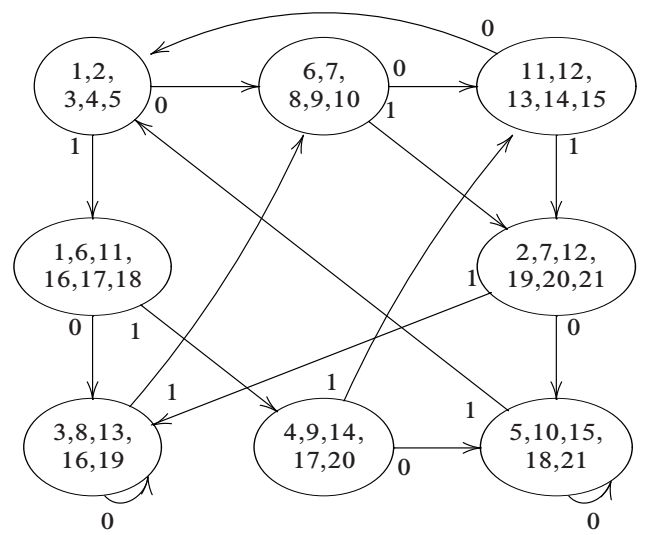

Рис. 16.

$$
\begin{array}{lll}
1 \stackrel{0}{\rightarrow} 8 \stackrel{0}{\rightarrow} 13 \stackrel{1}{\rightarrow} 7 \stackrel{1}{\rightarrow} 19 \stackrel{0}{\rightarrow} \varnothing & 2 \stackrel{0}{\rightarrow} 10 \stackrel{0}{\rightarrow} 15 \stackrel{0}{\rightarrow} 5 \stackrel{1}{\rightarrow} 1=\varnothing \\
3 \stackrel{1}{\rightarrow} 6 \stackrel{0}{\rightarrow} 13=\varnothing & 4 \stackrel{1}{\rightarrow} 11 \stackrel{0}{\rightarrow} 3=\varnothing & 9 \stackrel{1}{\rightarrow} 12 \stackrel{1}{\rightarrow} 19 \stackrel{0}{\rightarrow} \varnothing \\
14 \stackrel{1}{\rightarrow} 12=\varnothing & 16 \stackrel{1}{\rightarrow} 9=\varnothing & 17 \stackrel{1}{\rightarrow} 14=\varnothing \\
19 \stackrel{8}{\rightarrow} \varnothing & 20 \stackrel{1}{\rightarrow} 15=\varnothing & 21 \stackrel{1}{\rightarrow} 5=\varnothing
\end{array}
$$

Таким образом, система классов $Q_{i s}$ есть разбиение, поэтому система классов $\left(Q_{1}, Q_{2}, Q_{3}\right)$ есть разбиение, его диаграмма совпадает с диаграммой автомата $A$, при этом $r_{2} \neq f$. Случай рассмотрен.

Случай 5: $r_{1}=f^{-1}, r=h^{-1}$. Диаграмма классов $R_{i s}$ (рис. 17), и диаграмма пересечений (рис. 18) дают соотношения 


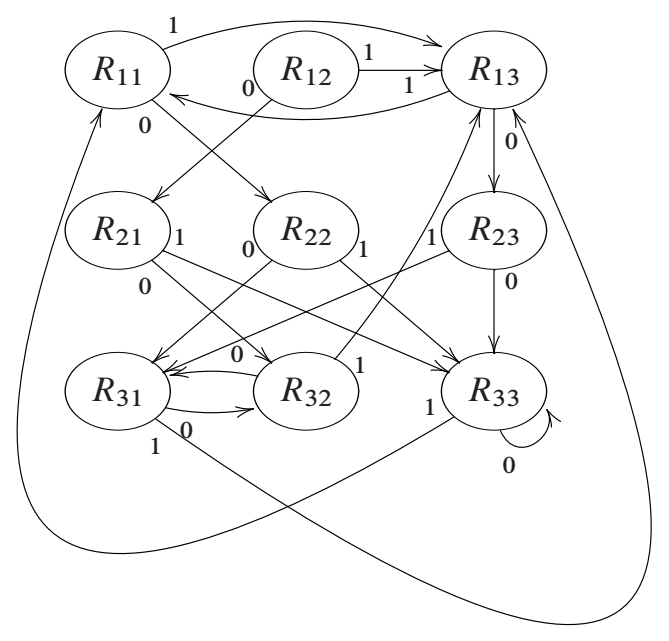

Рис. 17.

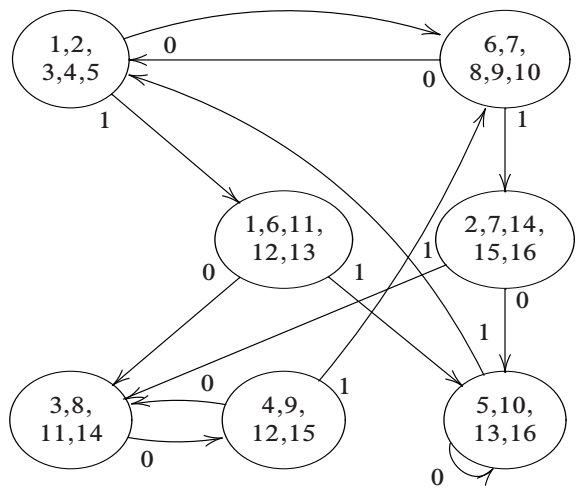

Рис. 18.
$1 \stackrel{1}{\rightarrow} 13 \stackrel{0}{\rightarrow} \varnothing$ $2 \stackrel{1}{\rightarrow} 11 \stackrel{0}{\rightarrow} \varnothing$ $3 \stackrel{0}{\rightarrow} 9 \stackrel{1}{\rightarrow} 7 \stackrel{1}{\rightarrow} 14 \stackrel{0}{\rightarrow} \varnothing$
$4 \stackrel{1}{\rightarrow} 6 \stackrel{0}{\rightarrow} 3=\varnothing \quad 5 \stackrel{1}{\rightarrow} 1=\varnothing \quad 8 \stackrel{0}{\rightarrow} 4=\varnothing$ $10 \stackrel{0}{\rightarrow} 5=\varnothing$
$12 \stackrel{1}{\rightarrow} 10=\varnothing$
$13 \stackrel{0}{\rightarrow} \varnothing$
$14 \stackrel{0}{\rightarrow} \varnothing$
$15 \stackrel{0}{\rightarrow} \varnothing$ $16 \stackrel{1}{\rightarrow} 3=\varnothing$

Система классов $Q_{i s}$ - разбиение, система классов $\left(Q_{1}, Q_{2}, Q_{3}\right)$ - разбиение, ее диаграмма совпадает с диаграммой автомата $A$, при этом $r_{2} \neq f$, что и требовалось доказать.

Случай 6: $r_{1}=f^{-1}, r=h$. Диаграмма классов $R_{i s}$ (рис. 19) и диаграмма пересечений (рис. 20) дают соотношения

$$
1 \stackrel{1}{\rightarrow} 20 \stackrel{0}{\rightarrow} \varnothing \quad 2 \stackrel{0}{\rightarrow} 10 \stackrel{1}{\rightarrow} 1=\varnothing \quad 3 \stackrel{0}{\rightarrow} 10=\varnothing \quad 4 \stackrel{0}{\rightarrow} 8 \stackrel{0}{\rightarrow} 15 \stackrel{1}{\rightarrow} 2=\varnothing
$$


Рис. 19.
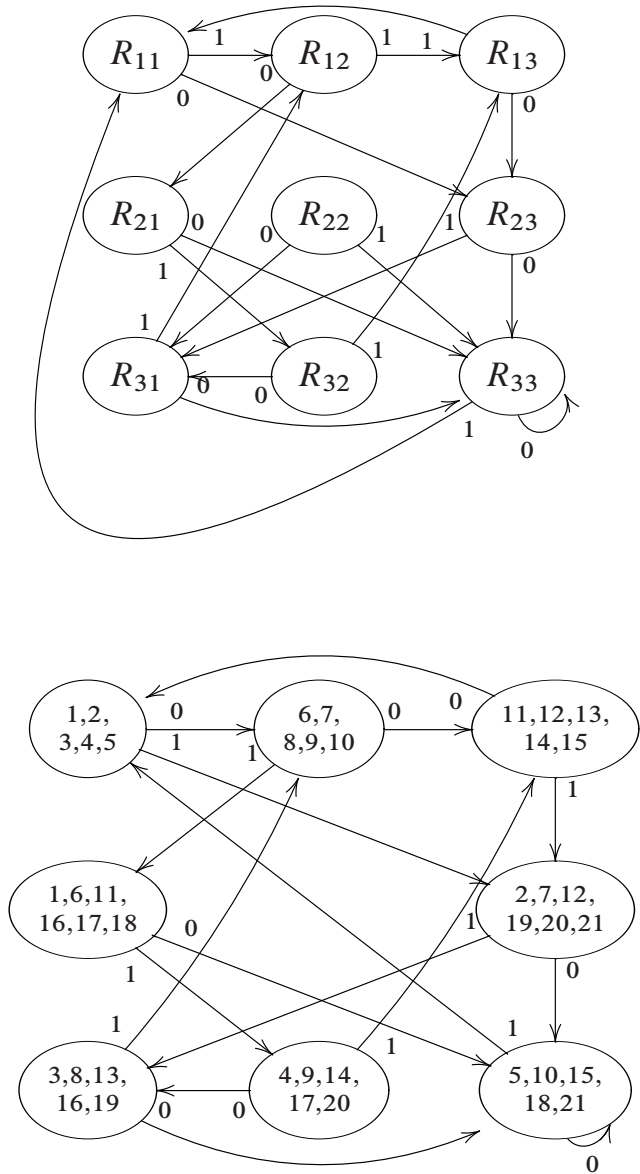

Рис. 20.
$5 \stackrel{1}{\rightarrow} 2=\varnothing \quad 6 \stackrel{0}{\rightarrow} 15=\varnothing$
$7 \stackrel{0}{\rightarrow} 15=\varnothing$
$9 \stackrel{0}{\rightarrow} 13 \stackrel{0}{\rightarrow} 5=\varnothing$
$11 \stackrel{0}{\rightarrow} 5=\varnothing$
$12 \stackrel{0}{\rightarrow} 5=\varnothing \quad 14 \stackrel{0}{\rightarrow} 3=\varnothing$
$16 \stackrel{1}{\rightarrow} 9=\varnothing \quad 17 \stackrel{0}{\rightarrow} \varnothing$
$18 \stackrel{1}{\rightarrow} 4=\varnothing$
$19 \stackrel{1}{\rightarrow} 8=\varnothing \quad 20 \stackrel{0}{\rightarrow} \varnothing$
$21 \stackrel{1}{\rightarrow} 3=\varnothing$

Таким образом, все пересечения пусты, система классов $\left(Q_{1}, Q_{2}, Q_{3}\right)$ - разбиение, ее диаграмма совпадает с диаграммой автомата $A$, при этом $r_{2} \neq f$, что и требовалось доказать.

Случай 7: $\quad r_{1}=h^{-} 1, r=h$. Диаграмма $R_{i s}$ (рис. 21) и диаграмма пересечений (рис. 22) дают соотношения 
Рис. 21.
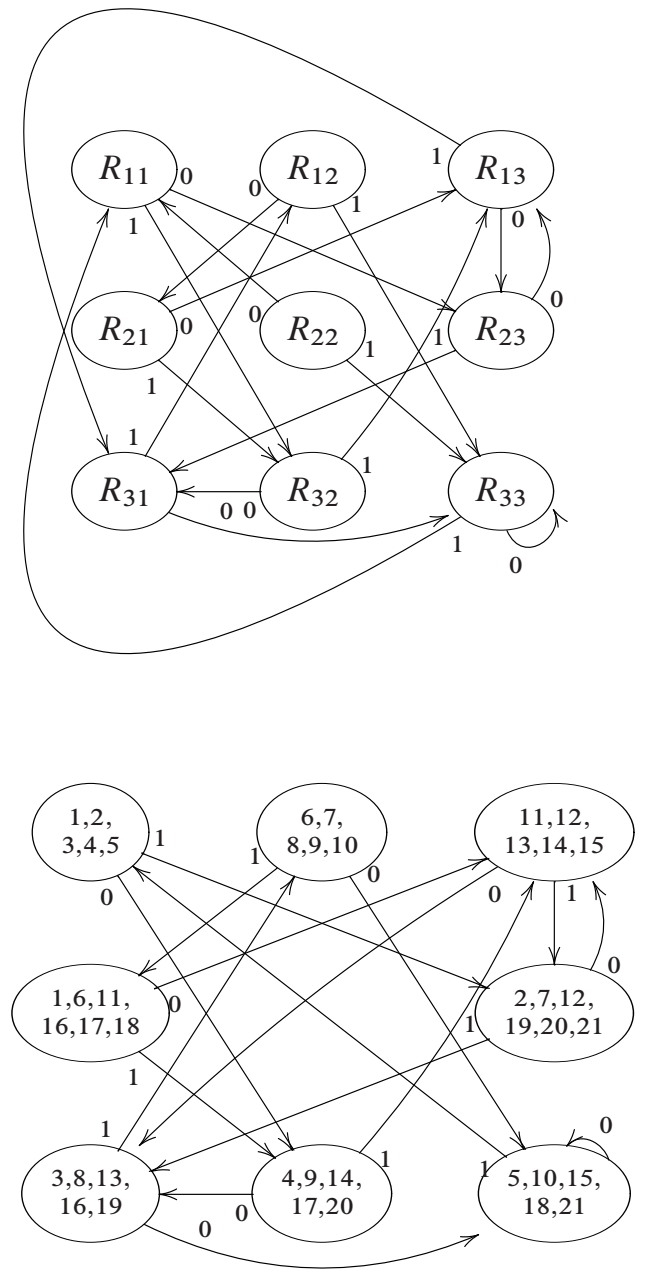

Рис. 22.

$$
\begin{array}{crlll}
1 \stackrel{0}{\rightarrow} 14 \stackrel{1}{\rightarrow} 12 \stackrel{0}{\rightarrow} 13 \stackrel{0}{\rightarrow} \varnothing & 2 \stackrel{0}{\rightarrow} 14=\varnothing & 3 \stackrel{0}{\rightarrow} \varnothing & 4 \stackrel{0}{\rightarrow} \varnothing & 5 \stackrel{0}{\rightarrow} \varnothing \\
6 \stackrel{0}{\rightarrow} 15 \stackrel{1}{\rightarrow} 2=\varnothing & 7 \stackrel{0}{\rightarrow} 15=\varnothing & 8 \stackrel{1}{\rightarrow} 6=\varnothing & 9 \stackrel{0}{\rightarrow} \varnothing & 10 \stackrel{1}{\rightarrow} 1=\varnothing \\
11 \stackrel{0}{\rightarrow} 13=\varnothing & 16 \stackrel{0}{\rightarrow} 15=\varnothing & 17 \stackrel{0}{\rightarrow} 13=\varnothing & 18 \stackrel{0}{\rightarrow} 15=\varnothing & \\
19 \stackrel{0}{\rightarrow} 15=\varnothing & 20 \stackrel{0}{\rightarrow} 13=\varnothing & 21 \stackrel{0}{\rightarrow} 15=\varnothing &
\end{array}
$$

Таким образом, снова имеем разбиение, диаграмма которого совпадает с диаграммой автомата $\bar{B}$, при этом $r_{2} \neq h$, и случай рассмотрен.

Случай 8: $\quad r_{1}=h^{-1}, r=f$. Диаграмма $R_{i s}$ (рис. 23) и диаграмма пересечений (рис. 24) дают соотношения 


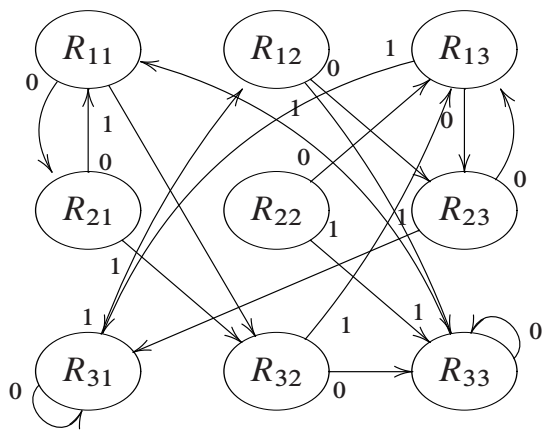

Рис. 23.

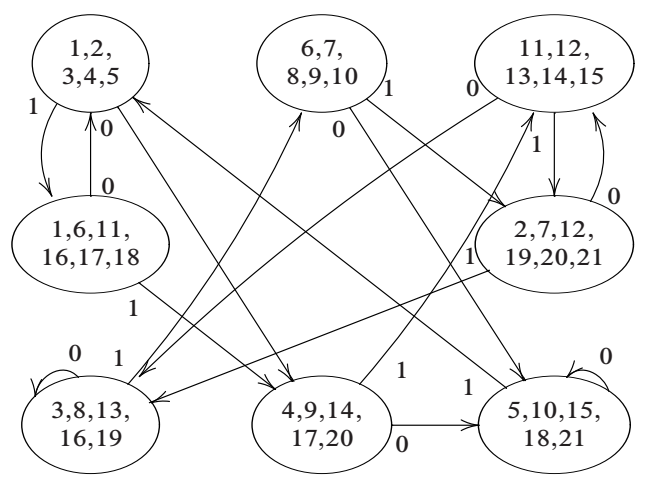

Рис. 24.

$$
\begin{array}{cclll}
1 \stackrel{0}{\rightarrow} 4 \stackrel{0}{\rightarrow} \varnothing & 2 \stackrel{0}{\rightarrow} 14 \stackrel{0}{\rightarrow} \varnothing & 3 \stackrel{0}{\rightarrow} \varnothing & 5 \stackrel{0}{\rightarrow} \varnothing & 6 \stackrel{0}{\rightarrow} 5=\varnothing \\
7 \stackrel{0}{\rightarrow} 15 \stackrel{0}{\rightarrow} \varnothing & 8 \stackrel{0}{\rightarrow} \varnothing & 9 \stackrel{1}{\rightarrow} 12 \stackrel{1}{\rightarrow} 19 \stackrel{1}{\rightarrow} 8=\varnothing & 10 \stackrel{1}{\rightarrow} 2=\varnothing & \\
11 \stackrel{0}{\rightarrow} 3=\varnothing & 13 \stackrel{1}{\rightarrow} 7=\varnothing & 14 \stackrel{0}{\rightarrow} \varnothing & 15 \stackrel{0}{\rightarrow} \varnothing & 16 \stackrel{0}{\rightarrow} 3=\varnothing \\
17 \stackrel{0}{\rightarrow} 5=\varnothing & 18 \stackrel{0}{\rightarrow} 5=\varnothing & 20 \stackrel{0}{\rightarrow} 15=\varnothing & 21 \stackrel{0}{\rightarrow} 15=\varnothing &
\end{array}
$$

Снова система $\left(Q_{1}, Q_{2}, Q_{3}\right)$ - разбиение с диаграммой $B$, и при этом $r_{2} \neq h$.

Случай 9: $r_{1}=h^{-1}, r=f^{-1}$. Диаграмма $R_{i s}$ (рис. 25) и диаграмма пересечений (рис. 26) дают соотношения

$$
\begin{array}{cclll}
1 \stackrel{0}{\rightarrow} 8 \stackrel{0}{\rightarrow} \varnothing & 2 \stackrel{0}{\rightarrow} 8=\varnothing & 3 \stackrel{0}{\rightarrow} \varnothing & 4 \stackrel{0}{\rightarrow} \varnothing & 5 \stackrel{0}{\rightarrow} \varnothing \\
6 \stackrel{0}{\rightarrow} 8=\varnothing & 7 \stackrel{0}{\rightarrow} 8=\varnothing & 9 \stackrel{0}{\rightarrow} \varnothing & 10 \stackrel{0}{\rightarrow} \varnothing & 11 \stackrel{0}{\rightarrow} 9=\varnothing \\
12 \stackrel{0}{\rightarrow} 10=\varnothing & 13 \stackrel{0}{\rightarrow} 10=\varnothing & 14 \stackrel{0}{\rightarrow} 9=\varnothing & 15 \stackrel{0}{\rightarrow} 10=\varnothing & 16 \stackrel{0}{\rightarrow} 10=\varnothing
\end{array}
$$

Снова справедливо разбиение $\left\{Q_{1}, Q_{2}, Q_{3}\right\}$. Случай рассмотрен. 


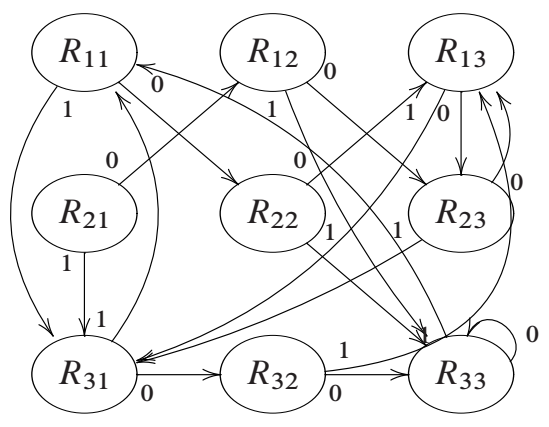

Pис. 25.

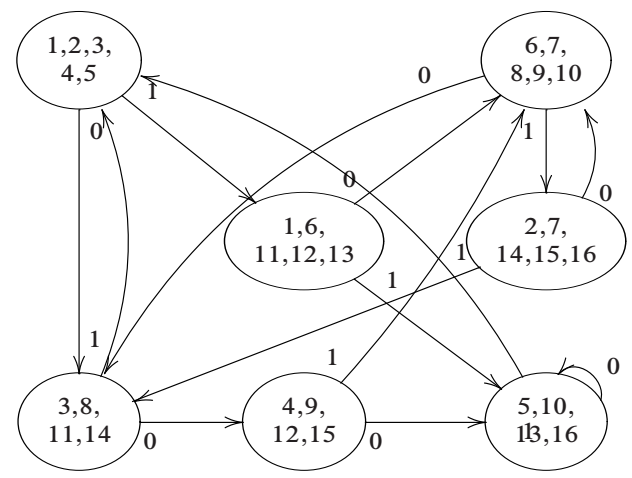

Рис. 26.

Случай 10: $r_{1}=h, r=f$. Диаграмма классов $R_{i s}$ (рис. 27) и диаграмма пересечений (рис. 28) дают соотношения

$$
\begin{array}{lll}
1 \stackrel{1}{\rightarrow} \varnothing & 2 \stackrel{1}{\rightarrow} \varnothing & 3 \stackrel{0}{\rightarrow} 14 \stackrel{0}{\rightarrow} 13 \stackrel{1}{\rightarrow} \varnothing \\
7 \stackrel{1}{\rightarrow} \varnothing & 8 \stackrel{0}{\rightarrow} 15 \stackrel{1}{\rightarrow} \varnothing \quad 9 \stackrel{1}{\rightarrow} \varnothing
\end{array}
$$

Каждый класс $Q_{i s}$ можно представить в виде $Q_{i s}=V_{i s} \cup \Pi_{i s}$, где

$$
\Pi_{i s}=\bigcup_{m, n}\left(Q_{i s} \cap Q_{m n}\right)
$$

то есть $V_{i s}-$ независимая часть $Q_{i s}$, а $\Pi_{i s}-$ состояния, каждое из которых входит и в $Q_{i s}$, и еще в какой-нибудь класс. Если из начального состояния $q$ 。 достижимы только состояния из множества $V$, то множество классов $V_{i s}$ образует разбиение множества $V$, и ситуация повторяет ранее рассмотренные случаи. Если из начального состояния достижимы состояния множества П, то в автомате $L$ имеется подавтомат, определяемый множеством состояний П, поскольку очевидно, что множество П замкнуто относительно действия функции переходов автомата $L$. Обозначим этот подавтомат через $L^{\circ}$. 


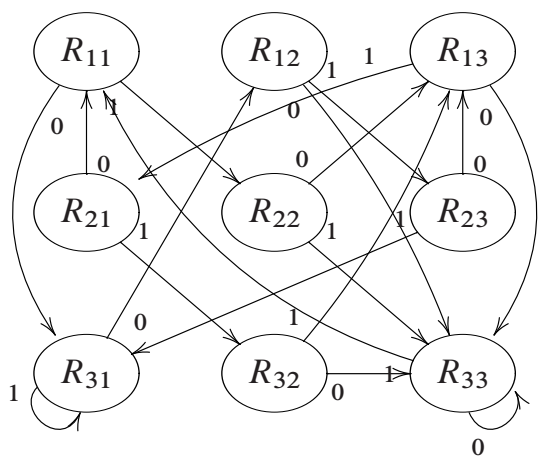

Рис. 27.

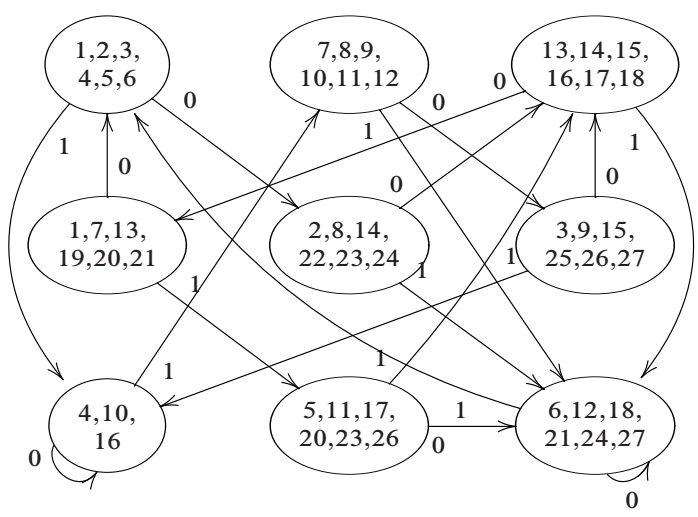

Рис. 28.

Рассмотрим классы

$$
\begin{aligned}
& K_{1}=\{4,5,6,10,11,12,16,17,18\}, \\
& K_{2}=\{19,20,21,22,23,24,25,26,27\},
\end{aligned}
$$

где номера 4, 5 и так далее приписаны в соответствии с диаграммой на рис. 28 соответствующим пересечениям классов.

Классы $K_{1}$ и $K_{2}$ не пересекаются, в противном случае хотя бы одно из пересечений $\{1,2,3,7,8,9,13,14,15\}$ оказалось бы непустым. Например, если $4 \cap 19 \neq \varnothing$, то $1 \neq \varnothing$.

Таким образом, пара классов $K_{1}, K_{2}$ образует разбиение множества П. Из диаграммы пересечений следует, что диаграмма этого разбиения совпадает с диаграммой автомата на рис. 5 , то есть это автомат, который мы обозначили через $D$. Нами показано, что автомат $D$ делит автомат $L$, поскольку у автомата $L$ нашелся подавтомат, который гомоморфен $D$.

Случай 11: $r_{1}=h, r=f^{-1}$. Диаграмма классов (рис. 29) и диаграмма пересечений (рис. 30) дают соотношения 


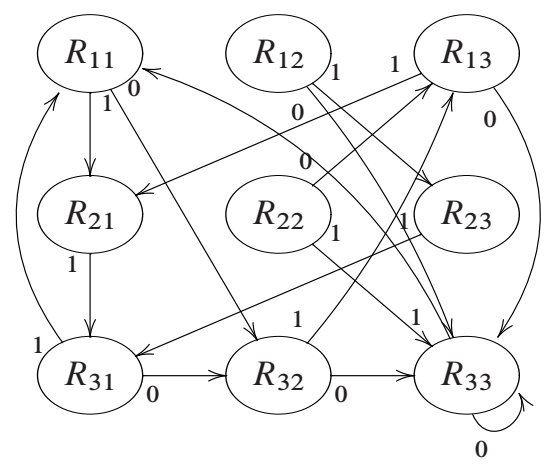

Рис. 29.

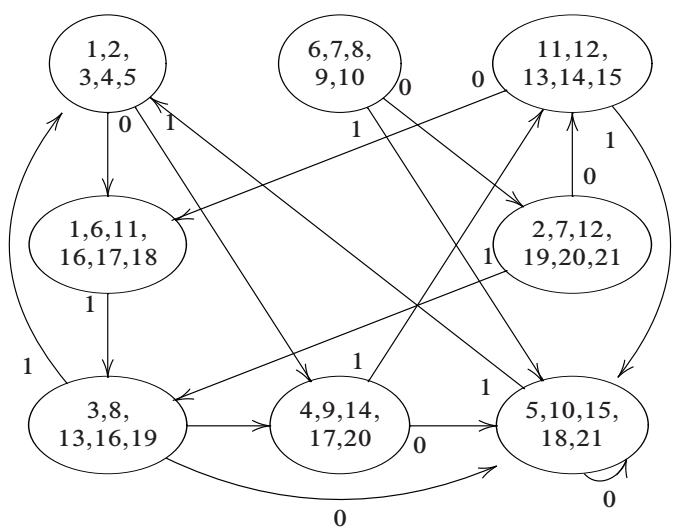

Рис. 30.

$$
\begin{array}{rrr}
1 \stackrel{1}{\rightarrow} \varnothing & 2 \stackrel{1}{\rightarrow} \varnothing \quad 6 \stackrel{1}{\rightarrow} \varnothing \quad 7 \stackrel{1}{\rightarrow} \varnothing \\
11 \stackrel{1}{\rightarrow} \varnothing \quad 12 \stackrel{1}{\rightarrow} \varnothing
\end{array}
$$

Снова имеем разбиение

$$
\begin{aligned}
& K_{1}=\{3,4,5,8,9,10,13,14,15\}, \\
& K_{2}=\{16,17,18,19,20,21\} .
\end{aligned}
$$

Диаграмма для этого разбиения совпадает с диаграммой автомата $D$. Случай рассмотрен.

Случай 12: $r_{1}=h, r=h^{-1}$. Диаграмма классов $R_{i s}$ (рис. 31) и диаграмма пересечений (рис. 32) дают соотношения 
Рис. 31.
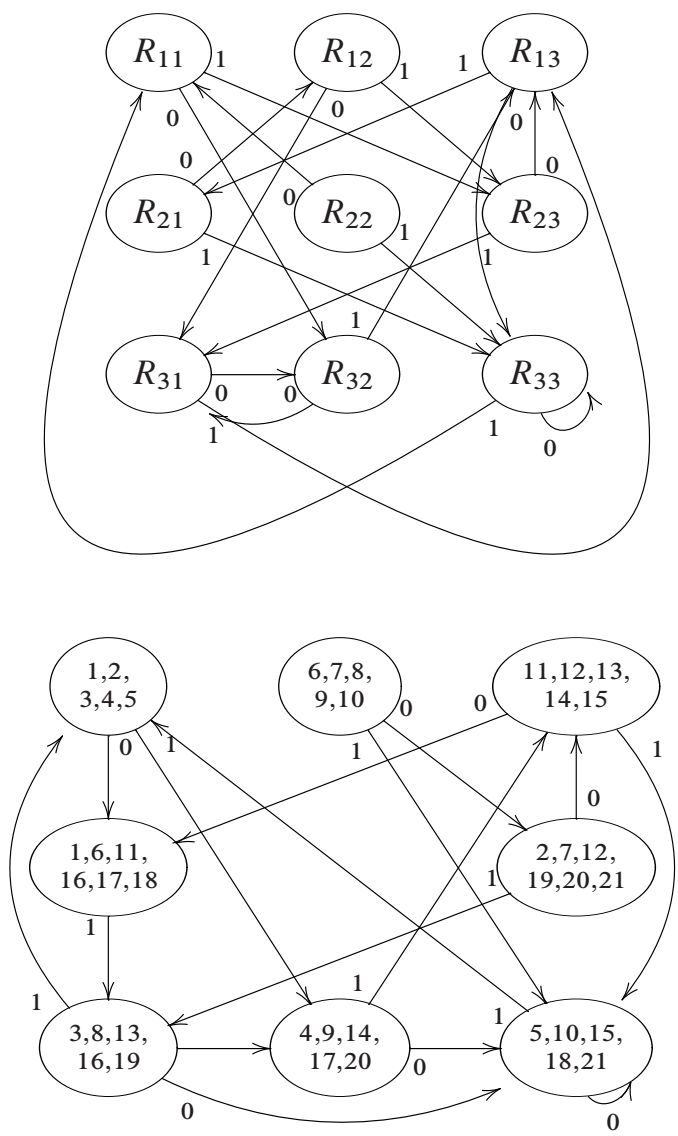

Рис. 32.

$$
\begin{array}{ll}
1 \stackrel{1}{\rightarrow} \varnothing & 2 \stackrel{1}{\rightarrow} \varnothing \\
1 \stackrel{1}{\rightarrow} \varnothing & 7 \stackrel{0}{\rightarrow} 12 \stackrel{1}{\rightarrow} \varnothing \\
11 \stackrel{0}{\rightarrow} 6=\varnothing &
\end{array}
$$

Снова имеем разбиение $\{3,4,5,8,9,10,13,14,15\},\{16,17,18,19,20,21\}$ на два класса. Диаграмма разбиения совпадает с диаграммой $D$. Случай рассмотрен.

Таким образом, случаи 10, 11 и 12 приводят нас к ситуации $r_{2} \neq h^{-1}$, и автомат, реализующий слово $r_{2} r_{3} \ldots r_{s}$, делится на $D$.

Случай 12a. Пусть $r_{2}=f$. Соответствующие диаграммы для классов $R_{i s}$ и диаграмма пересечений, представленные на рис. 33 и 34, дают соотношения

$$
\begin{array}{lll}
1 \stackrel{1}{\rightarrow} 5 \stackrel{0}{\rightarrow} 8 \stackrel{0}{\rightarrow} \varnothing & 2 \stackrel{0}{\rightarrow} 8=\varnothing \quad 3 \stackrel{0}{\rightarrow} 2=\varnothing \\
4 \stackrel{0}{\rightarrow} 8=\varnothing & 6 \stackrel{0}{\rightarrow} 7 \stackrel{1}{\rightarrow} 2=\varnothing
\end{array}
$$




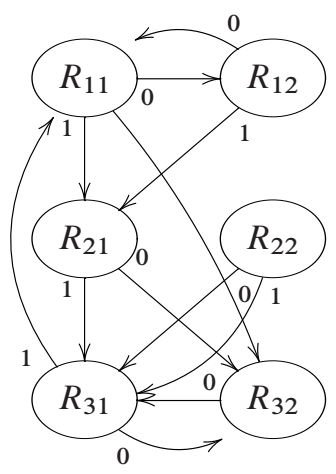

Рис. 33.

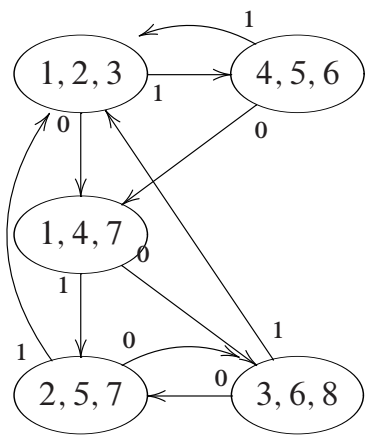

Рис. 34.

Следовательно, система $Q_{i s}$ - разбиение на множестве состояний автомата $L^{\circ}$, и система $Q_{1}, Q_{2}, Q_{3}$ - разбиение с диаграммой, совпадающей с диаграммой автомата $f^{-1}$, то есть слово $r_{3} r_{4} \ldots r_{s}$ обладает $S$-свойством.

Случай 12b. Если $r_{2}=f^{-1}$, то соответствующие диаграммы (рис. 35 и 36) дают соотношения

$$
\begin{aligned}
& 1 \stackrel{1}{\rightarrow} 4 \stackrel{0}{\rightarrow} \varnothing \quad 2 \stackrel{1}{\rightarrow} 1=\varnothing \quad 3 \stackrel{1}{\rightarrow} 1=\varnothing \\
& 5 \stackrel{1}{\rightarrow} 2=\varnothing,
\end{aligned}
$$

то есть получаем разбиение с диаграммой, совпадающей с диаграммой автомата $A$, следовательно, слово $r_{3} r_{4} \ldots r_{s}$ обладает $S$-свойством.

Случай 12c: $r_{2}=h$. Теперь получаем диаграммы, изображенные на рис. 37, 38. Диаграмма пересечений показывает, что $1 \stackrel{1}{\rightarrow} \varnothing$, и что множество пересечений $2,3,5,6,7,8$ замкнуто относительно функции переходов автомата $H$, реализующего слово $r_{3} r_{4} \ldots r_{s}$. Для этих множеств справедлива представленная на рис. 39 диаграмма. 


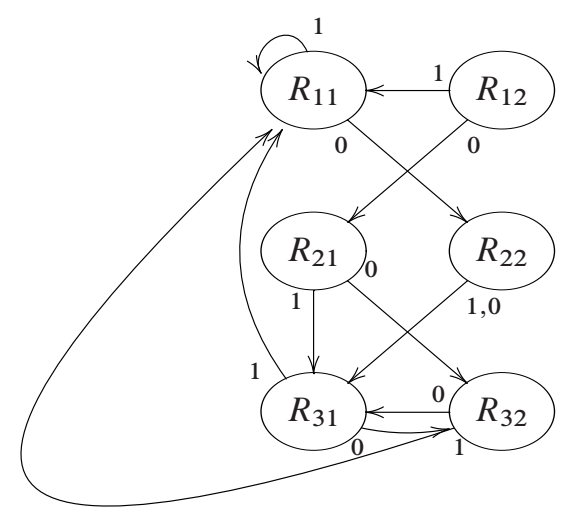

Рис. 35.

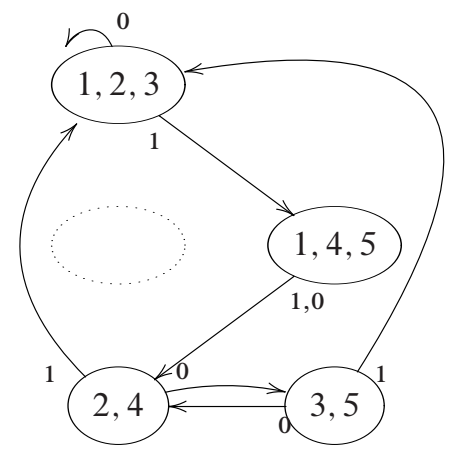

Рис. 36.

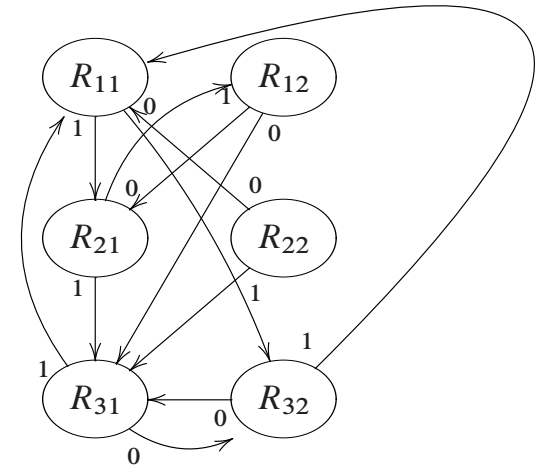

Рис. 37. 


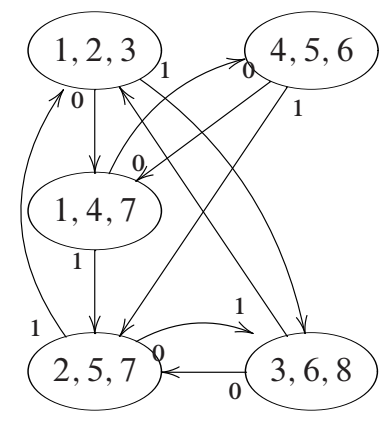

Рис. 38.

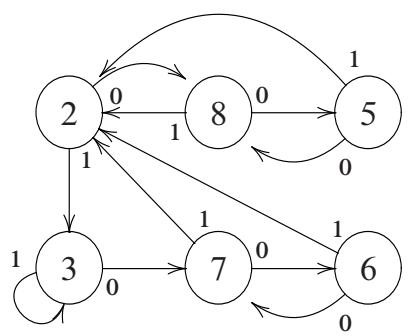

Рис. 39.

Обозначим этот подавтомат автомата $H$ через $H^{\circ}$. Набор подмножеств $2,3,5,6,7,8$ состояний автомата $H^{\circ}$ не является разбиением, однако, как следует из диаграммы на рис. 38 , имеет место равенство $5 \cap 8=\varnothing$. Пусть $r_{3}=f^{-1}$.

Аналогично предыдущим построениям, пусть $V-$ приведенный автомат, задающий слово $r_{4} r_{5} \ldots r_{s}$. Рассмотрим множество $H_{i k}, i \in\{1,2,3\}, k \in\{2,3,5,6,7,8\}$, классов состояний автомата $H$, таких что $W \in H_{i k}$, если в суперпозиции $\bar{A} V$ пара $(i, k)$ задает состояние $W$. С учетом диаграммы на рис. 39 , для классов $H_{i k}$ имеет место диаграмма переходов на рис. 40. Пусть $V_{i k}$ - множество состояний автомата $V$ такое, что $v \in V_{i k}$, если $(i, v) \in H_{i k}$. Тогда для него справедлива диаграмма на рис. 41 .

Важно, что из выше приведенного свойства $5 \cap 8=\varnothing$ следует, что $V_{38} \cap V_{35}=\varnothing$. Построим диаграмму пересечений для классов $V_{i k}$ (рис. 42), ее граф совпадает с графом диаграммы на рис. 41.

Имеют место соотношения

$$
\begin{aligned}
& 1 \stackrel{1}{\rightarrow} 15 \stackrel{0}{\rightarrow}\left(V_{38} \cap V_{35}\right)=\varnothing \quad 2 \stackrel{1}{\rightarrow} 15=\varnothing \quad 3 \stackrel{0}{\rightarrow} 11 \stackrel{1}{\rightarrow} 2=\varnothing \quad 22 \stackrel{1}{\rightarrow} 3=\varnothing \\
& 4 \stackrel{1}{\rightarrow} 1=\varnothing \quad 5 \stackrel{1}{\rightarrow} 1=\varnothing \quad 6 \stackrel{1}{\rightarrow} 1=\varnothing \quad 7 \stackrel{1}{\rightarrow} 1=\varnothing \quad 13 \stackrel{1}{\rightarrow} 2=\varnothing \quad 23 \stackrel{1}{\rightarrow} 3=\varnothing \\
& 8 \stackrel{0}{\rightarrow} 12 \stackrel{0}{\rightarrow} 11=\varnothing \quad 9 \stackrel{0}{\rightarrow} 14 \stackrel{1}{\rightarrow} 2=\varnothing \quad 10 \stackrel{0}{\rightarrow} 11=\varnothing \quad 21 \stackrel{1}{\rightarrow} 3=\varnothing \\
& 16 \stackrel{1}{\rightarrow} 3=\varnothing \quad 17 \stackrel{1}{\rightarrow} 3=\varnothing \quad 18 \stackrel{1}{\rightarrow} 3=\varnothing \quad 19 \stackrel{1}{\rightarrow} 3=\varnothing \quad 20 \stackrel{1}{\rightarrow} 10=\varnothing \quad 24 \stackrel{1}{\rightarrow} 3=\varnothing
\end{aligned}
$$

Следовательно, множество классов $V_{i k}$ образует разбиение множества состояний $\left\{\bigcup_{i, k} V_{i k}\right\}=\widetilde{V}$ автомата $V$. Соответственно, тройка $V_{1}, V_{2}, V_{3}$ классов $V_{i}=\bigcup_{k} V_{i k}$ также образует разбиение множества $\tilde{V}$. При этом диаграмма разбиения совпадает с диа- 


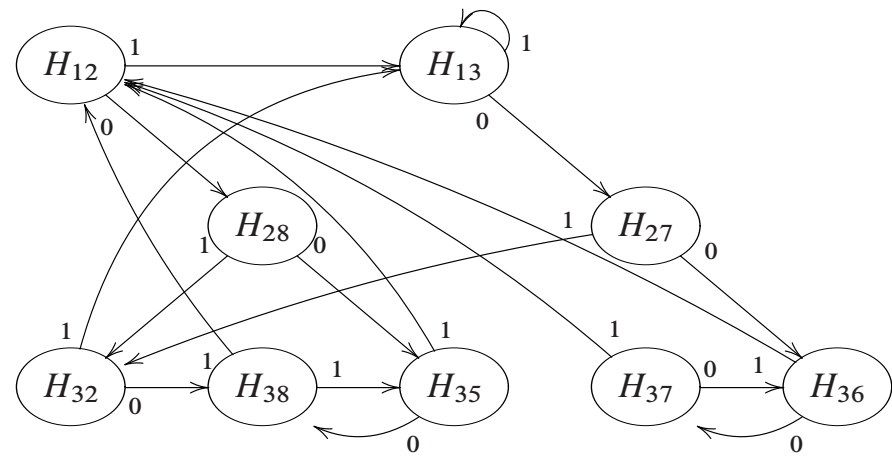

Рис. 40.

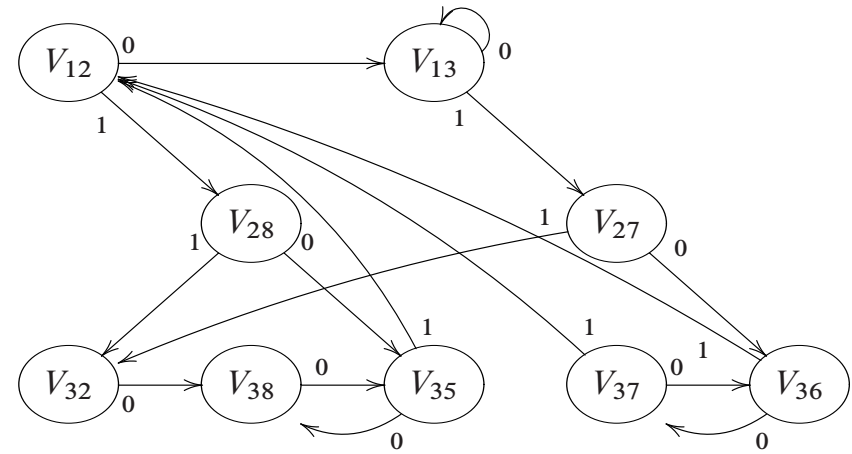

Рис. 41.

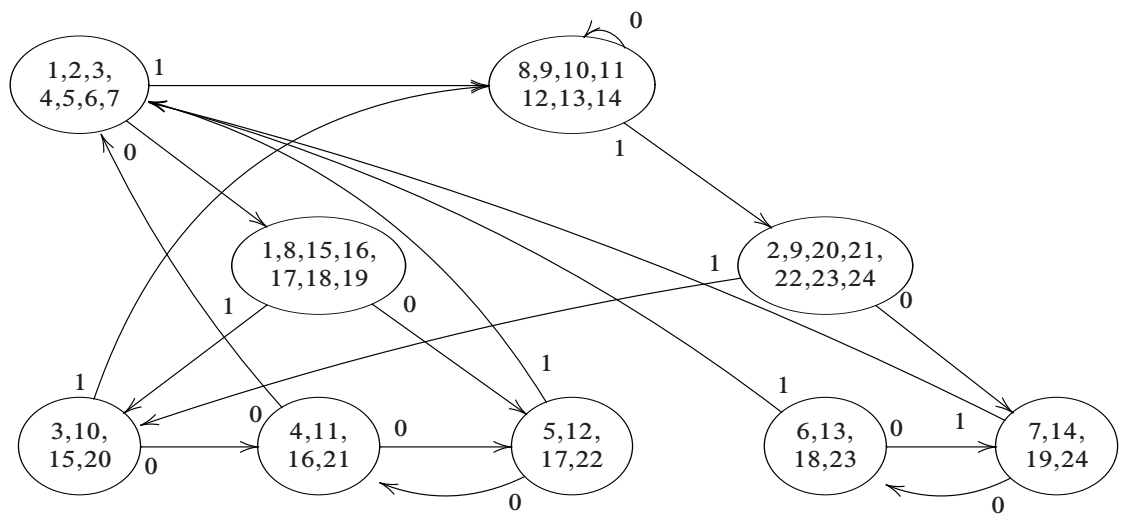

Рис. 42. 


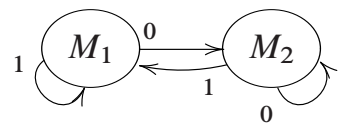

Рис. 43.

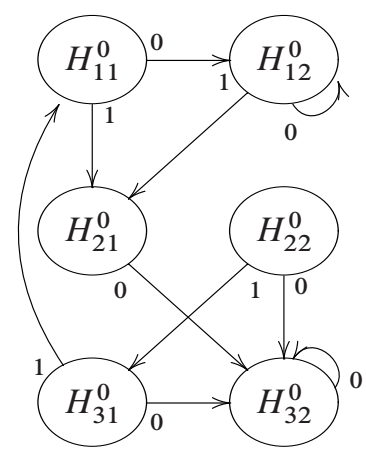

Рис. 44.

граммой автомата $A$. Следовательно, слово $r_{4} r_{5} \ldots r_{s}$ обладает $S$-свойством.

Нам осталось рассмотреть случаи $r_{3}=f, r_{3}=h$. Для этого заметим, что пара множеств $M_{1}, M_{2}$ состояний автомата $H^{\circ}, M_{1}=\{2,3\}, M_{2}=\{5,6,7,8\}$, образует разбиение множества состояний $H^{\circ}$. В самом деле, из диаграммы пересечений на рис. 38 следует, что $2 \cap 5=\varnothing$ (они на одной горизонтали), аналогично, $2 \cap 6=\varnothing, 2 \cap 8=\varnothing$. Так как $1=\varnothing$, мы видим, что $2 \cap 7=\varnothing$. Аналогично находим, что $3 \cap 5=\varnothing, 3 \cap 6=\varnothing$, $3 \cap 7=\varnothing, 3 \cap 8=\varnothing$.

Диаграмма для этой пары представлена на рис. 43. Она совпадает с диаграммой автомата $G$. Таким образом, $G$ делит $H^{\circ}$.

Пусть теперь $r_{3}=f$. Соответствующие диаграммы классов $H_{i k}^{\circ}$ и диаграмма пересечений представлены на рис. 44 и рис. 45 . Справедливы соотношения

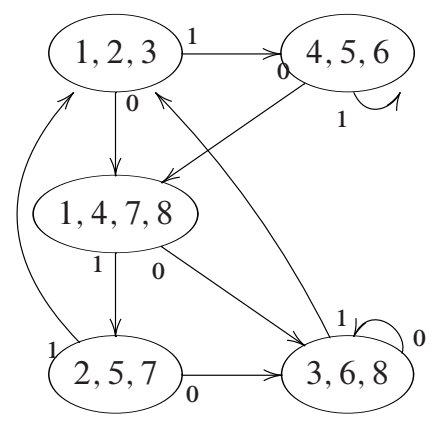

Рис. 45. 


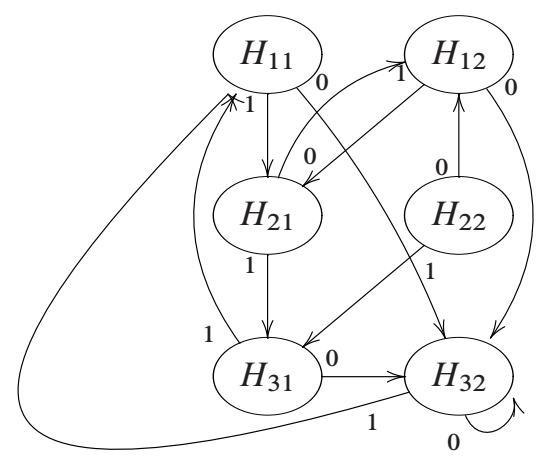

Рис. 46.

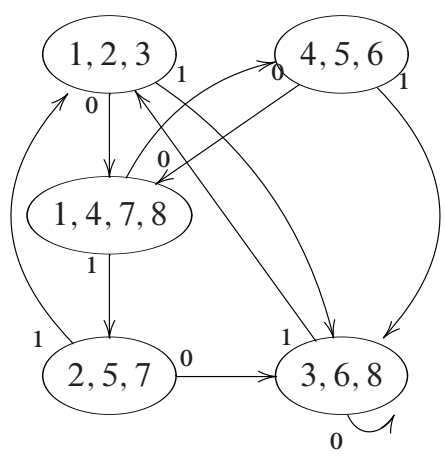

Рис. 47.

$$
\begin{array}{ll}
1 \stackrel{0}{\rightarrow} 8 \stackrel{1}{\rightarrow} 2 \stackrel{1}{\rightarrow} \varnothing \quad 3 \stackrel{1}{\rightarrow} \varnothing \quad 4 \stackrel{1}{\rightarrow} 5 \stackrel{1}{\rightarrow} \varnothing \\
6 \stackrel{1}{\rightarrow} \varnothing & 7 \stackrel{1}{\rightarrow} 2=\varnothing
\end{array}
$$

Таким образом, все пересечения пусты, тройка $V_{1}, V_{2}, V_{3}$ - это разбиение множества состояний $V$, и слово $r_{4} r_{5} \ldots r_{s}$ обладает $S$-свойством.

Наконец, пусть теперь $r_{3}=h$. Диаграмма $H_{i k}$ (рис. 46) и диаграмма пересечений (рис. 47) дают соотношения

$$
1 \stackrel{1}{\rightarrow} \varnothing \quad 4 \stackrel{1}{\rightarrow} \varnothing
$$

Пара классов

$$
M_{1}=\{2,3,5,6\}, \quad M_{2}=\{7,8\}
$$

образует разбиение множества состояний $V$. Диаграмма переходов для этой пары совпадает с диаграммой $D$. Слово $r_{4} r_{5} \ldots r_{s}$ не начинается с $h^{-1}$ и $D$ делит $V$, что возвращает нас к ситуации после случаев $10,11,12$. Индуктивно рассуждая, проходим по всему слову $R$. Лемма доказана. 
Теперь мы можем доказать следующую теорему.

Теорема 1. Подгруппа $A S_{2}$, порожденная элементами $\left\{f, f^{-1}, h, h^{-1}\right\}$, является свободной группой.

Доказательство. Предположим, что в алфавите $\left\{f, f^{-1}, h, h^{-1}\right\}$ существует правильное слово, такое, что $r_{1} r_{2} \ldots r_{s} r_{s+1}=1$. Можно считать, что $r_{1} \neq r_{s+1}^{-1}$, в противном случае рассмотрим слово $r_{2} r_{3} \ldots r_{s}$, которое также равно 1 . Из равенства $r_{1} r_{2} \cdots r_{s} r_{s+1}=1$ следует равенство $r_{1} r_{2} \cdots r_{s}=r_{s+1}^{-1}$. Отсюда следует, что автомат, реализующий слово $r_{1} r_{2} \ldots r_{s}$, гомоморфен (даже равен) автомату, реализующему $r_{s+1}^{-1}$, и при этом $r_{1} \neq r_{s+1}^{-1}$. Следовательно, слово $r_{1} r_{2} \ldots r_{s}$ обладает $S$-свойством. Индуктивно применяя лемму 1 , мы получим, что либо слово $r_{s}$ обладает $S$-свойством, тогда один из автоматов $\left\{f, f^{-1}, h, h^{-1}\right\}$ делит другой, неравный ему автомат из этого списка, либо автомат $D$ делит автомат, реализующий $r_{s}$, либо автомат $G$ делит автомат, реализующий $r_{s}$. Все эти случаи приводят к противоречию. Теорема доказана.

\section{Список литературы}

1. Кудрявцев В. Б., Алешин С. В., Подколзин А. С., Введение в теорию автоматов. Наука, Москва, 1985.

2. Каргаполов М. И., Мерзляков Ю. И., Основы теории групn. Наука, Москва, 1982.

3. Алешин С. А., Свободная группа конечных автоматов. Вестник Московского унив., сер. I (1983), № 4, 12-14.

4. Vorobets M., Vorobets Y., On a free group of transformations defined by an automaton. Geom. Dedicata (2007) 124, 237-249.

5. Żuk A., Groupes engendrés par les automates. Astérisque (2008) 317, 141-174.

6. Bondarenko I, Grigorchuk R., Kravchenko R., Muntyan Y., Nekrashevych V., Savchuk D., Šunić, Z., On classification of groups generated by 3-state automata over a 2-letter alphabet. Algebra Discrete Math. (2008), № 1, 1-163. 